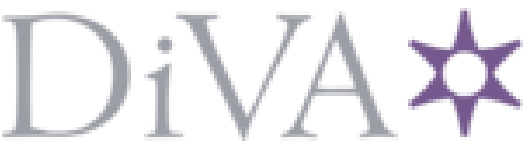

http://www.diva-portal.org

Postprint

This is the accepted version of a paper presented at The 11th International Conference on Communications COMM2O16.

Citation for the original published paper:

Yao, Y., Popescu, A. (2016)

On Energy Consumption in Mobile Multimedia Networks with OpwnFlow Switch. In:

N.B. When citing this work, cite the original published paper.

Permanent link to this version:

http://urn.kb.se/resolve?urn=urn:nbn:se:bth-15639 


\title{
On Energy Consumption in Mobile Multimedia Networks with OpenFlow Switch
}

\author{
Yong Yao and Adrian Popescu \\ Blekinge Institute of Technology, 37179 Karlskrona, Sweden \\ Email: \{yong.yao, adrian.popescu\}@bth.se
}

\begin{abstract}
In mobile multimedia networks, the video flow usually operates in an end-to-end manner from Head-End to mobile terminals. However, measuring the energy consumption associated with a video flow is a sophisticated process due to the complexity related to this. In the paper, a theoretical measurement approach is suggested to estimate the energy consumption of a video flow through mobile multimedia networks enhanced with the support of OpenFlow switch. Two different power models are built up to compute the traffic related energy consumption at the network element side. The numerical derivation of these two theoretical models is presented.
\end{abstract}

Index Terms-Mobile multimedia network, energy consumption, Software Defined Network, OpenFlow switch.

\section{INTRODUCTION}

With the advance of new wireless technologies and ubiquitous radio access, mobile multimedia is becoming a very important application for both service providers and end users. This also leads to different business models such as mobile television, mobile conferencing, and remote gaming. A Mobile Multimedia Network (MMN) generally refers to several parts, which are known as the video contribution, the network distribution and the mobile terminals. The video contribution is connected to the functions of generating and processing the video content, which is eventually transferred onto the network for further distribution to mobile terminals.

However, designing efficient MMN is a sophisticated process due to requirements for the provision of sufficient Quality of Service (QoS) and Quality of Experience (QoE) required by terminals. Moreover, a very challenging problem raises in the form of demand for reducing the power consumption in IPbased video networks [1], [2]. Connected to this problem, one needs to model and to measure the energy consumption for the end-to-end (e2e) video flows through the particular network elements. This can be done by using different methodologies like theoretical studies, simulation experiments and practical measurements in real networks. There is a large amount of work done along with this research line. For instance, the authors of [3] report on a theoretical approach to determine the so-called absolute energy efficiency for telecommunication network systems. In [4], the authors suggest a measurement based model to estimate the power consumption of the network elements. The corresponding estimation also considers the energy consumed by the network traffic through the network element. In [5], the authors report on the results of energy consumption measured at the smartphone side in the case of using camera to record and stream video. In [6] the author addresses the need of a balance between the energy efficiency and performance in the cloud based data center. The author therefore suggests to employ Software Defined Network (SDN) and Network Function Virtualization (NFV) to achieve the addressed balance. Clearly, the focus of these studies is laid on the energy consumption of the particular networking elements. To the best of our knowledge, there are few studies done so far on the energy consumption of video flow in MMN. The video flow is defined to be an e2e data transmission from the video streaming source side (e.g., Head-End) to the video consumer side (e.g., mobile terminals).

Typically, a MMN is composed of various network components in terms of hardware, software and network solutions. Examples of hardware are Head-End, routers, switches, WiFi access points and mobile terminals. Important software and network solutions are, e.g., video encoding/decoding algorithms, routing algorithms, edge-cloud, SDN. The diversity of the network components indicates the complexity in practically measuring the energy consumption of video flow in MMN. To solve this problem, we suggest a new theoretical model to estimate the energy consumption of video flow along a particular e2e routing path in MMN. Further, this model includes two parts. They are the power model for traditional networking elements like, e.g., switches, routers, and the power model for OpenFlow switch.

The rest of paper is as follows. Section II describes the system model. The networking power model is built up in Section III. The OpenFlow Switch power model for SDN is presented in Section IV. Finally, we conclude the paper in Section V.

\section{SySTEM MODEL}

Given a particular MMN, we consider a video flow consisting of four main elements, as shown in Fig. 1. They are the video data center (VDC), the transport entity, the delivery entity and the video consumers. Their detailed descriptions are as follows:

- Video Data Center (VDC): this is responsible for streaming the video data to video consumers. For instance, for both OTT and IPTV models, a key component of VDC is the Head-End.

- Transport: network elements between the Head-End and the delivery part of the network

- Delivery: it is related to the particular network solution that can (efficiently) deliver the video data from VDC 


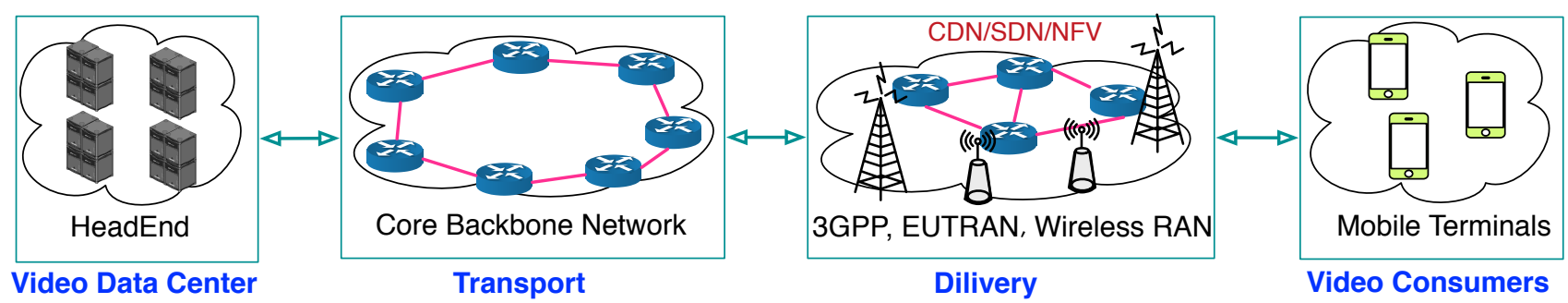

Fig. 1. Typical e2e video flow through mobile multimedia network.

to video consumers. For example, a suitable network solution for delivering the video data in the OTT model is the CDN.

- Video Consumers: they are connected to various terminal devices (e.g., mobiles, SetTopBox) that consume the video data.

Moreover, a video flow is usually associated with a particular video streaming scenario (e.g., mobile television, mobile cloud gaming), which may prescribe a group of specific requirements for the practical implementation. These implementation requirements can be further defined with respect to different parameters like, e.g., QoE, QoS, power consumption.

\section{Networking Power Model}

\section{A. Networking System Model}

Based on the above-described MMN, a video flow is considered with $M$ devices labelled with $d_{1}, d_{2}, \ldots, d_{M}$, respectively. Further, $N$ different traffic flows are assumed to exist in the networking system in a particular temporal frame. These traffic flows are labelled with $f_{1}, f_{2}, \ldots, f_{N}$, respectively. The main difference among them is in terms of different packet sizes. A particular traffic flow $f_{n}$ is assumed to have a number $v_{n}$ of packets, where each packet is assumed to have a uniform size $l_{n}$, where $n \in\{1,2, \ldots, N\}$. In the following, a simple transmission scenario is considered, which means that each of $N$ traffic flows is assumed to be transmitted through each of $M$ devices only once.

Further, a particular time interval $[t, t+\Delta t]$ is considered to be partitioned into $K$ identical unit time slots (e.g., per second). Each time slot has an uniform length $\delta$, where $\Delta t=$ $K \delta$. Further, let $t_{k}$ denote a particular time moment $(t+\delta k)$ where $k \in\{1,2, \ldots, K\}$. The e2e energy consumption of the considered networking system during the interval $[t, t+\Delta t]$ is calculated as follows.

\section{B. Traffic related power consumption model for individual devices}

Let $P\left(m, t_{k}\right)$ denote the power consumption (expressed in, e.g., Watts or Joules/sec) measured on device $d_{m}$ during the time slot $\left[t_{k}, t_{k+1}\right]$, where $m \in\{1,2, \ldots, M\}$ and $t_{k}>0$. Typically, $P\left(m, t_{k}\right)$ can be expressed as the sum of two parts of power consumptions. The first part is the power used for device $d_{m}$ under the situation of no traffic flow through it, i.e., the so-called idle power and it is denoted by $P_{\text {idle }}\left(m, t_{k}\right)$. The second part is the power used for transmitting data during the unit time interval (e.g., per second), i.e., the so-called traffic related power and it is denoted by $P_{\text {traf }}\left(m, t_{k}\right)$. The total power consumption is therefore:

$$
P\left(m, t_{k}\right)=P_{\text {idle }}\left(m, t_{k}\right)+P_{\text {traf }}\left(m, t_{k}\right)
$$

Information about the idle power of devices can be obtained from testing experiments or the corresponding manufacturing companies provide it. Hence, the focus of e2e power consumption is laid on the traffic related power of devices.

Considering the time slot $\left[t_{k}, t_{k+1}\right]$, the power used by device $d_{m}$ to deal with traffic flow $f_{n}$ is denoted by $P_{\text {traf }}^{(n)}\left(m, t_{k}\right)$. From the packet transmission point of view, $P_{\text {traf }}^{(n)}\left(m, t_{k}\right)$ is related to two different operations on the traffic flow $f_{n}$. They are: i) storing and forwarding data (i.e., in terms of multiple packets), and ii) processing packets. The corresponding power consumptions are denoted by $P_{S \& F}^{(n)}\left(m, t_{k}\right)$ and $P_{P r o}^{(n)}\left(m, t_{k}\right)$. That means:

$$
P_{\text {traf }}^{(n)}\left(m, t_{k}\right)=P_{S \& F}^{(n)}\left(m, t_{k}\right)+P_{P r_{o}}^{(n)}\left(m, t_{k}\right)
$$

By considering all $N$ different traffic flows transmitted through the device $d_{m}$, the total traffic related power consumption during the time slot $\left[t_{k}, t_{k+1}\right]$ is given by:

$$
P_{\text {Traf }}^{(n)}\left(m, t_{k}\right)=\sum_{n=1}^{N}\left[P_{S \& F}^{(n)}\left(m, t_{k}\right)+P_{\text {Pro }}^{(n)}\left(m, t_{k}\right)\right]
$$

\section{Traffic related energy consumption model for individual} devices

The idle power of device $d_{m}$ is assumed to be a constant value during an unit time $\left[t_{k}, t_{k+1}\right]$, e.g., a second. Accordingly, let $E_{\text {idle }}\left(m, t_{k}\right)$ denote the energy consumption (expressed in, e.g., Joules) associated with the idle power of device $d_{m}$. This is calculated by:

$$
E_{\text {idle }}\left(m, t_{k}\right)=\delta P_{\text {idle }}\left(m, t_{k}\right)
$$

Regarding the energy consumption used for dealing with traffic flows, the corresponding formulation is, however, more complicated. This is because the above-mentioned two operations have different responsibilities, which lead to different ways for quantifying the energy consumptions. Specifically, the energy used for storing and forwarding packets is associated to the size of data transmitted in a time slot. While, the energy used for processing packets is associated with the number of packets transmitted in a time slot. 
During the time slot $\left[t_{k}, t_{k+1}\right]$, let $E_{S \& F}^{(n)}\left(m, t_{k}\right)$ denote the energy consumption used by device $d_{m}$ to store and forward traffic flow $f_{n}$, and let $E_{P r o}^{(n)}\left(m, t_{k}\right)$ denote the energy consumption for processing packets. With regard to the wellknow equation $P($ Watts $)=E($ Joules $/$ bit $) R($ bits $/$ sec $)$, they satisfy the two equations below:

$$
\left\{\begin{array}{l}
P_{S \& F}^{(n)}\left(m, t_{k}\right)=E_{S \& F}^{(n)}\left(m, t_{k}\right) \alpha^{(n)}\left(m, t_{k}\right) \\
P_{P P_{0}}^{(n)}\left(m, t_{k}\right)=\left[E_{P \text { Pro }_{0}}^{(n)}\left(m, t_{k}\right) / l_{n}\right] \alpha^{(n)}\left(m, t_{k}\right)
\end{array}\right.
$$

where, $\alpha^{(n)}\left(m, t_{k}\right)$ is defined to be the data transmission throughput at device $d_{m}$ during the time slot $\left[t_{k}, t_{k+1}\right]$. For instance, if the time slot $\left[t_{k}, t_{k+1}\right]$ is equal to one second, the metric used for measuring $\alpha^{(n)}\left(m, t_{k}\right)$ is given by bits/sec. These two equations create two different metrics for measuring the energy consumption used by device $d_{m}$ to deal with traffic flows $f_{n}$. They are the so-called incremental energy per bit and incremental energy per packet, which are denoted by $E_{\text {Incre }}^{(n, b i t)}\left(m, t_{k}\right)$ and $E_{\text {Incre }}^{(n, p k t)}\left(m, t_{k}\right)$, respectively. They are computed by:

$$
\left\{\begin{array}{l}
E_{\text {Incre }}^{(n, b i t)}\left(m, t_{k}\right)=E_{S \& F}^{(n)}\left(m, t_{k}\right)+E_{\text {Pro }}^{(n)}\left(m, t_{k}\right) / l_{n} \\
E_{\text {Incre }}^{(n, p k t)}\left(m, t_{k}\right)=l_{n} E_{S \& F}^{(n)}\left(m, t_{k}\right)+E_{\text {Pro }}^{(n)}\left(m, t_{k}\right)
\end{array}\right.
$$

For simplicity purposes, the second metric incremental energy per packet is used in the following, together with a new metric defined as the packet rate (i.e., packets/sec). This metric is denoted by $\beta^{(n)}\left(m, t_{k}\right)$, and it satisfies:

$$
\beta^{(n)}\left(m, t_{k}\right)=\alpha^{(n)}\left(m, t_{k}\right) / l_{n}
$$

By considering all $N$ different traffic flows, the total traffic related energy consumption at device $d_{m}$ during time slot $\left[t_{k}, t_{k+1}\right]$ is:

$$
\begin{aligned}
& P_{\text {Traf }}\left(m, t_{k}\right) \\
= & \sum_{n=1}^{N}\left[P_{S \& F}^{(n)}\left(m, t_{k}\right)+P_{P r o}^{(n)}\left(m, t_{k}\right)\right] \\
= & \sum_{n=1}^{N}\left[E_{\text {Incre }}^{(n, p k t)}\left(m, t_{k}\right) \beta^{(n)}\left(m, t_{k}\right)\right]
\end{aligned}
$$

Note that, in the work reported by [4], the authors assume that they can obtain the information about the packet length of all traffic flows through each device $d_{m}$. They simplify the above calculation as follows:

$$
\sum_{n=1}^{N}\left[l_{n} \beta^{(n)}\left(m, t_{k}\right)\right] \simeq \bar{l} \sum_{n=1} N \beta^{(n)}\left(m, t_{k}\right)=\bar{l} \tilde{\beta}\left(m, t_{k}\right)
$$

where $\bar{l}$ and $\tilde{\beta}\left(m, t_{k}\right)$ are the mean packet length and the total packet rate of all $N$ traffic flows, respectively. However, this simplification may not be applicable in reality. This is because it is extremely difficult to measure the packet lengths and rates of all traffics passing through all devices or equipments deployed in the real networking system. The above-simplified calculation is therefore not adopted, and the focus is laid instead on the e2e energy consumption of the individual traffic flow.
D. Traffic related e2e energy consumption model for a networking system

Let $\tilde{P}_{\text {Traf }}^{(n)}\left(t_{k}\right)$ denote the total power consumption used by all devices to deal with the traffic flow $f_{n}$ during the time slot $\left[t_{k}, t_{k+1}\right]$. This is calculated with:

$$
\tilde{P}_{\text {Traf }}^{(n)}\left(t_{k}\right)=\sum_{m=1}^{M}\left[E_{\text {Incre }}^{(n, p k t)}\left(m, t_{k}\right) \beta^{(n)}\left(m, t_{k}\right)\right]
$$

For the time interval $[t, t+\Delta t]$, where $\Delta t=K \delta$, let $\tilde{E}_{\text {Traf }}^{n}(\Delta t)$ denote the total energy consumption used by all $N$ devices to deal with the traffic flow $f_{n}$. This is given by:

$$
\begin{aligned}
& \tilde{E}_{\text {Traf }}^{(n)}(\Delta t) \\
= & \sum_{k=1}^{K} \delta \tilde{P}_{\text {Traf }}^{(n)}\left(t_{k}\right) \\
= & \sum_{k=1}^{K} \sum_{m=1}^{M}\left[E_{\text {Incre }}^{(n, p k t)}\left(m, t_{k}\right) \beta^{(n)}\left(m, t_{k}\right)\right] \\
\stackrel{\Rightarrow}{=} \quad & \sum_{m=1}^{M}\left\{\int_{m=1}^{M}\left[E_{\text {Incre }}^{(n, p k t)}(m, \tau) \beta^{(n)}(m, \tau) d \tau\right]\right\}
\end{aligned}
$$

Furthermore, we assume that, for traffic flow $f_{n}$, the incremental energy per packet $E_{\text {Incre }}^{(n, p k t)}(m, \tau)$ at device $d_{m}$ does not change during the time interval $\left[t_{k}, t_{k+1}\right]$. In other words, we have $E_{\text {Incre }}^{(n, p k t)}(m, \tau)=\bar{E}_{\text {Incre }}^{(n, p k t)}(m)$. This assumption is applicable under different cases, e.g., when there is no traffic congestion experienced by the device $d_{m}$.

We finally obtain the total energy consumption used by all devices to deal with the traffic flow:

$$
\tilde{E}_{\text {Traf }}^{(n)}(\Delta t)=\sum_{m=1} M\left\{\bar{E}_{\text {Incre }}^{(n, p k t)}(m) \int_{\tau=t}^{t+\Delta t} \beta^{(n)}(m, \tau) d \tau\right\}
$$

This formula refers to the variable part in the power consumption profile for an e2e chain of equipments traversed by a traffic flow. This is also known as the power consumption due to packet forwarding. The total e2e energy consumption is therefore given by the sum, taken over all $M$ devices in the e2e route, of individual energy consumptions. Furthermore, the energy consumption of an individual device is given by the product of incremental energy per packet multiplied by the packet rate.

\section{OpenFlow Switch Power Model}

Software Defined Networking (SDN) is defined to be an architecture where the control and data planes are decoupled, network intelligence and state are logically centralized, and the underlying network infrastructure is abstracted from the applications [8]. There are open interfaces between the devices in the control plane and those in the data plane as well. A key motivation for SDN is to abstract the control plane from the data plane, i.e., to separate the control from the packet forwarding. This motivation also gives rise to the need of supporting programmability for related hardware

\section{A. OpenFlow Switch}

OpenFlow is a protocol defined between the forwarding elements of the network and the control elements of SDN architecture [9]. It allows direct access to the control entities 
for the forwarding elements of network devices, e.g., routers and switches. OpenFlow defines a data plane abstraction of the forwarding devices (e.g., switches), and also provides an API or protocol to them. In a particular OpenFlow switch, a forwarding element is specifically abstracted as a flow table, which is applied by the Match+Action principle (Fig. 2).

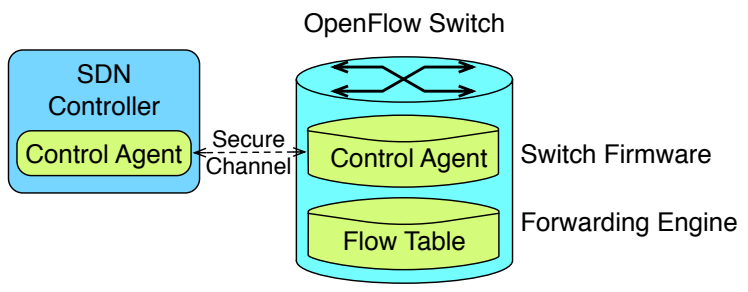

Fig. 2. SDN controller and OpenFlow switch.

Basically, the OpenFlow specifies an instruction set to manage the flow table from a remote controller like, e.g., in the case of an IP router or Ethernet switch. This means that one needs to model the traffic related energy consumption for the OpenFlow switch.

\section{B. Traffic related power consumption model for OpenFlow switch}

We assume that the device $d_{m} \in\left\{d_{1}, d_{2}, \ldots, d_{M}\right\}$ is an OpenFlow switch. During a time slot $\left[t_{k}, t_{k}+\Delta t\right]$, the power used by this device to deal with traffic flow $f_{n} \in$ $\left\{f_{1}, f_{2}, \ldots, f_{N}\right\}$ is denoted by $\left.P_{\text {Traf }}^{*}(n), t_{k}\right)$. Differing from the formulation presented in subsections III-B and III-C, $P_{\text {Traf }}^{*}\left(n, t_{k}\right)$ consists of three parts of power consumptions. These parts are used for storing/forwarding, processing packets and carrying out the above-mentioned Match+Action progress. They are denoted by $P_{S \& F}^{*}(n)\left(m, t_{k}\right), P_{P r o}^{*}(n)\left(m, t_{k}\right)$ and $P_{M \& A}^{*(n)}\left(m, t_{k}\right)$, respectively. One therefore obtains:

$P_{\text {Traf }}^{*}(n)\left(m, t_{k}\right)=P_{S \& F}^{*}(n)\left(m, t_{k}\right)+P_{P r o}^{*}(n)\left(m, t_{k}\right)+P_{M \& A}^{*(n)}\left(m, t_{k}\right)$

\section{Traffic related energy consumption model for OpenFlow switch}

With regard to subsection III-C, the energy consumptions for storing/forwarding and processing packets can be accordingly computed. In the following, the focus is laid on the energy consumption for carrying out the Match+Action progress for a unit time $\left[t_{k}, t_{k}+\Delta t\right]$, which is denoted by $E_{M \& A}^{*(n)}\left(m, t_{k}\right)$. Similar to the calculation of energy consumption of processing a packet, $P_{M \& A}^{*(n)}\left(m, t_{k}\right)$ and $E_{M \& A}^{*}(n)\left(m, t_{k}\right)$ satisfy the following:

$$
P_{M \& A}^{*(n)}\left(m, t_{k}\right)=\left[E_{M \& A}^{*(n)}\left(m, t_{k}\right) / l_{n}\right] \alpha^{(n)}\left(m, t_{k}\right)
$$

where $\alpha^{(n)}\left(m, t_{k}\right)$ denotes the data transmission throughput at device $d_{m}$ during the time slot $\left[t_{k}, t_{k+1}\right]$, and denotes the uniform packet size of the traffic flow, where the packets of the traffic $f_{n}$ has an uniform size $l_{n}$.
Regarding the traffic related e2e energy consumption model for multiple OpenFlow switches, the corresponding formulation can be developed based on the above-derived formula and the formulation presented in subsection III-C.

\section{Energy saving with using SDN}

Compared to a normal switch (i.e., without supporting SDN functionality), an OpenFlow switch may consume more energy due to the Match+Action progress. However, there are many benefits of using SDN. This is because the SDN can dynamically adjust network elements to save energy, e.g., by activating/deactivating devices on a need-basis, reprogramming the involved OpenFlow switches depending on energy-saving needs/models, dynamic bandwidth allocation/scheduling for resource optimization.

\section{CONClusion}

Mobile multimedia communication with the support of OpenFlow Switch is considered. The energy consumption of the video flow along the e2e routing path is studied. A theoretical measurement approach is advanced to estimate the video traffic related energy consumption along the e2e routing path. Specifically, two different power models are suggested for traditional networking elements and OpenFlow switches, respectively. Future works are to conduct numerical performance analysis based on the suggested power models, and to carry out the experimental experiment to validate the analysis results.

\section{ACKNOWLEDGMENT}

This work is supported by the European Celtic-Plus project CONVINcE and was partially funded by Finland, France, Sweden and Turkey.

\section{REFERENCES}

[1] European Commission, Horizon 2020 Energy Efficiency, http://ec.europa.eu/easme/en/horizon-2020-energy-efficiency, December, 2015.

[2] A. Popescu, "Greening of IP-Based Video Distribution Networks: Developments and Challenges", IEEE 10th International Conference on Communications, Bucharest, May 2014.

[3] M. Parker and S. Walker, "Roadmapping ICT: An Absolute Energy Efficiency Metric", IEEE/OSA Journal of Optical Communications and Networking, vol. 3, no. 8, pp. A49-A58, 2011.

[4] Center for Energy Efficient Telecommunications, University of Melbourne, "Measurement based network element power modeling", TREND/GreenTouch Joint Workshop on Green and Energy Efficient Networking, Turin, 2013.

[5] S.V. Rajaraman, M. Siekkinen, and M.A. Hoque, "Energy Consumption Anatomy of Live Video Streaming from a Smartphone", IEEE 25th International Symposium on Personal, Indoor and Mobile Radio Communications, Washington DC, 2014.

[6] C. Kolias, "Software-defined networking and network functions virtualization (NFV) in energy-efficient data centers.", 2nd International Workshop on Energy-Efficient Data Centres, Berkeley, CA, May 2013.

[7] CONVINcE: Consumption Optimization in Video Networks, CelticPlus project, https://www.celticplus.eu/project-convince/, September, 2014.

[8] Open Network Foundation, "Software-Defined Networking: The New Norm for Networks", ONF White Paper, April, 2012.

[9] D. Kreutz, F.M.V. Ramos, P.E Veríssimo, C.E. Rothenberg, S. Azodolmolky, and S. Uhlig, "Software-defined networking and network functions virtualization (NFV) in energy-efficient data centers", Proceedings of the IEEE, vol. 103, no. 1, pp. 14-76, 2015. 